\title{
Taking Automated Mineralogy from the Lab into the Field
}

\author{
C. Lang \& M. Hiscock \\ Oxford Instruments NanoAnalysis, Halifax Road, High Wycombe, HP12 3SE, UK.
}

This paper reports on an experimental system that employs an automated mineralogy system on a tabletop scanning electron microscope.

SEM-EDS based automated mineralogy is widely used in the geological industries in order to study mineral deposits, for mine planning and for oil and gas reservoir analysis. It is also being increasingly used in academic settings to study phase distributions and the compositions of bulk samples. Several solutions integrating SEM and EDS hardware with dedicated software are available on the market. Systems have been installed at oil drilling sites and mining sites employing ruggedized SEM designs to withstand transport requirements and local environmental conditions. However, none of the automated mineralogy systems available today are truly portable and therefore of little use to the field geologist. In other application areas such as the forensics and biological sciences, however, small footprint tabletop or desktop SEMs are becoming increasingly popular [1].

Most table-top SEMs in use today are mainly used as high-resolution imaging tools with some offering integrated EDS detectors to provide some analytical capability. In comparison, dedicated automated mineralogy systems rely on extremely fast analytical performance, often incorporating several EDS detectors in order to reach the analysis speeds required. In order to build an automated mineralogy system based on a table-top SEM as the imaging and analysis platform, the software needs to be designed to minimize the reliance on fast EDS acquisition and maximize the information gained from the electron image.

Most table-top microscopes enable the acquisition of backscattered electron images which contain significant elemental contrast. By utilizing the BSE image to identify where different mineral phases are located and to determine where EDS analysis should be performed, a tabletop microscope can be optimized for automated mineralogy use.

Here, a tabletop microscope equipped with an Oxford Instruments SDD EDS system and INCAMineral software was used to perform an automated mineralogical analysis across the large surface area of a particulated geological sample which has undergone mineral liberation processing. Initially, a limited number of fields of view were analysed with EDS spectra acquired from all grains in order to characterise the sample and determine which mineral phases were represented by each BSE image grey level range. Once this information had been obtained it was then possible to perform a run where information was gathered solely from the BSE image with mineral phases associated with each grey level threshold. From this data, information on the degree of liberation and association of phases for the sample was determined. This data could in turn be used to optimize the liberation process.

This work demonstrates the possibility of performing automated mineralogy on a tabletop microscope. Hence, investigations of geological samples can take place in locations where it may not be possible to gain immediate access to a dedicated system but where it may be possible for a smaller, more easily moved system to be installed, potentially on a more temporary basis. Such locations could include the 
mine site or field bases. By optimizing mineral liberation analysis software for the capabilities of the tabletop microscope, valuable information can be automatically obtained in an efficient way.
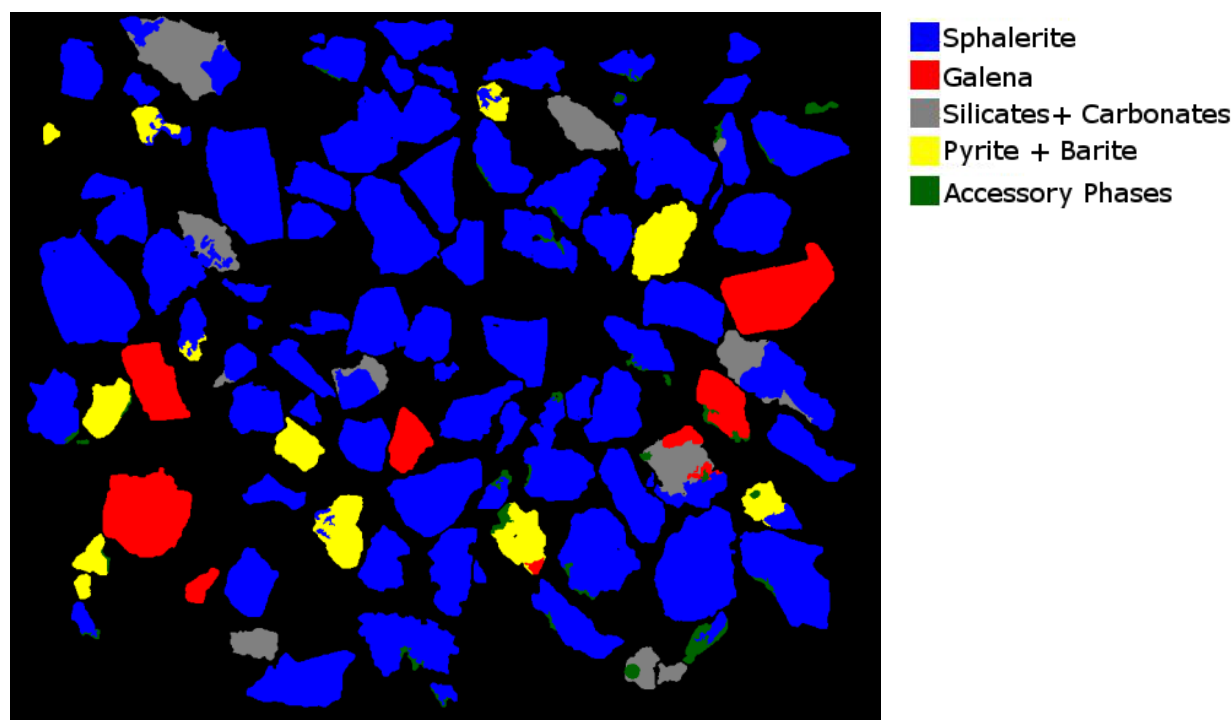

Figure 1. Mineral phase image of sample analysed entirely with EDS.
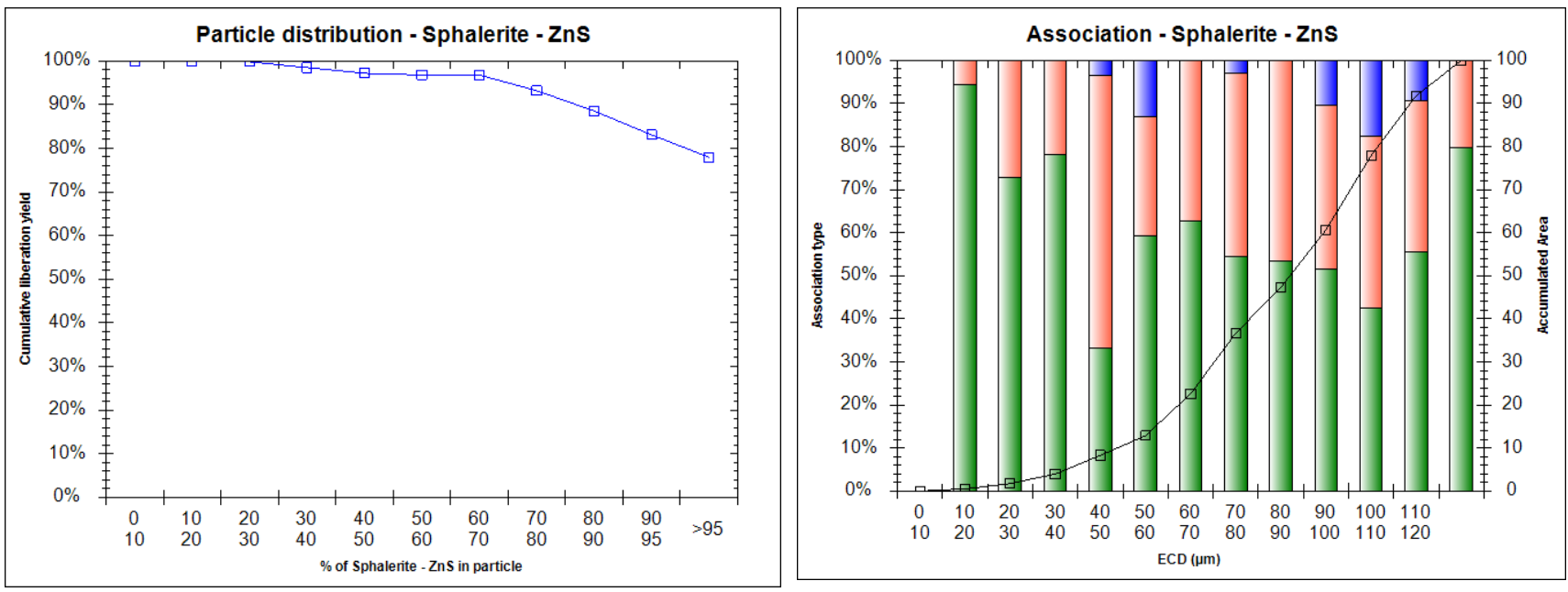

Figure 2. Liberation and association data determined for sphalerite.

In association diagram, green refers to solo association, red to binary and blue to ternary.

\section{References}

[1] K Linse \& P Marks "Tabletop Electron Microscopy at the British Antarctic Survey" (Lab Asia) January/February 2013 